Swarthmore College

Works

6-1-2006

\title{
Fast High Resolution Echelle Spectroscopy Of A Laboratory Plasma
}

C. D. Cothran

Jerome Fung , '06

Michael R. Brown

Swarthmore College, doc@swarthmore.edu

M. J. Schaffer

Follow this and additional works at: https://works.swarthmore.edu/fac-physics

Part of the Physics Commons

Let us know how access to these works benefits you

\section{Recommended Citation}

C. D. Cothran; Jerome Fung , '06; Michael R. Brown; and M. J. Schaffer. (2006). "Fast High Resolution Echelle Spectroscopy Of A Laboratory Plasma". Review Of Scientific Instruments. Volume 77, Issue 6. DOI: 10.1063/1.2212405

https://works.swarthmore.edu/fac-physics/97

This work is brought to you for free by Swarthmore College Libraries' Works. It has been accepted for inclusion in Physics \& Astronomy Faculty Works by an authorized administrator of Works. For more information, please contact myworks@swarthmore.edu. 


\section{AIP $\mid \begin{aligned} & \text { Review of } \\ & \text { Scientifici nstruments }\end{aligned}$}

\section{Fast high resolution echelle spectroscopy of a laboratory plasma}

C. D. Cothran, J. Fung, M. R. Brown, and M. J. Schaffer

Citation: Review of Scientific Instruments 77, 063504 (2006); doi: 10.1063/1.2212405

View online: http://dx.doi.org/10.1063/1.2212405

View Table of Contents: http://scitation.aip.org/content/aip/journal/rsi/77/6?ver=pdfcov

Published by the AIP Publishing

Articles you may be interested in

High-throughput charge exchange recombination spectroscopy system on MAST

Rev. Sci. Instrum. 77, 10F131 (2006); 10.1063/1.2354309

Measurements of impurity spectra using UV/visible spectroscopic system in a GAMMA 10 plasma

Rev. Sci. Instrum. 77, 10F103 (2006); 10.1063/1.2227441

High resolution transmission grating spectrometer for edge toroidal rotation measurements of tokamak plasmas Rev. Sci. Instrum. 75, 4165 (2004); 10.1063/1.1789591

Telecentric viewing system for light collection from a z-pinch plasma

Rev. Sci. Instrum. 72, 2224 (2001); 10.1063/1.1353188

Fast charged-coupled device spectrometry using zoom-wavelength optics

Rev. Sci. Instrum. 68, 1015 (1997); 10.1063/1.1147924

\section{ZABER}

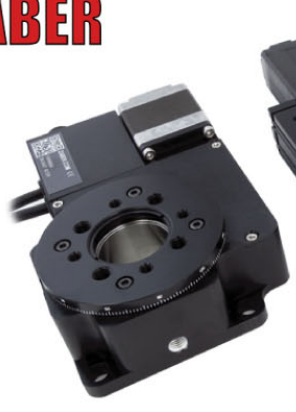

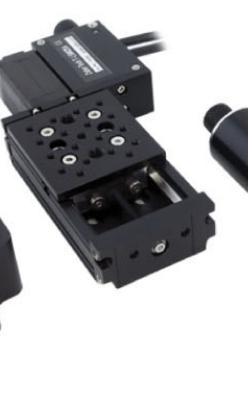

Automate your research applications with Zaber's line of high precision positioning devices.

Low cost. Built-in controllers. Simple to set up and easy to use.

Learn more at zaber.com 


\title{
Fast high resolution echelle spectroscopy of a laboratory plasma
}

\author{
C. D. Cothran, ${ }^{\text {a) }}$ J. Fung, and M. R. Brown \\ Department of Physics and Astronomy, Swarthmore College, Swarthmore, Pennsylvania 19081
}

M. J. Schaffer

General Atomics, P.O. Box 85608, San Diego, California 92186

(Received 22 February 2006; accepted 13 May 2006; published online 21 June 2006)

An echelle diffraction grating and a multianode photomultiplier tube are paired to construct a high resolution $\left(R=\lambda / \delta \lambda \approx 2.5 \times 10^{4}\right)$ spectrograph with fast time response for use from the UV through the visible. This instrument has analyzed the line shape of C III impurity ion emission at $229.687 \mathrm{~nm}$ over the lifetime $(\approx 100 \mu$ s) of the hydrogen plasmas produced at SSX. The ion temperature and line of sight average velocity are inferred from the observed thermal broadening and Doppler shift of the line. The time resolution of these measurements is about $1 \mu \mathrm{s}$, sufficient to observe the fastest magnetohydrodynamic activity. (C) 2006 American Institute of Physics. [DOI: 10.1063/1.2212405]

\section{INTRODUCTION}

Numerous spectroscopic techniques exist for characterizing laboratory plasmas (see Hutchinson ${ }^{1}$ ). Perhaps the most widely used method is the measurement of ion temperature and line of sight average fluid velocity based on the thermal Doppler broadening and Doppler shift of an emission line from an impurity ion species. There are many recent examples of the application of this basic technique, commonly referred to as ion Doppler spectroscopy (IDS). The Maryland Centrifugal Experiment (MCX) has used IDS to measure supersonic toroidal $E \times B$ flow which may provide centrifugal confinement in a magnetic mirror device., ${ }^{2,3}$ Radially sheared axial flow which stabilizes the kink mode in a $Z$ pinch has been observed at the $\mathrm{ZaP}$ experiment. ${ }^{4,5}$ IDS measurements of rapid ion heating have been correlated with magnetic relaxation events in the Madison Symmetric Torus (MST) ${ }^{6,7}$ Many other examples of the utility of IDS for examining the dynamics of laboratory plasmas can easily be found. ${ }^{8,9}$

This article describes a fast time response IDS instrument designed to investigate radial and toroidal (azimuthal) midplane flows and ion heating during counterhelicity spheromak merging experiments ${ }^{10}$ at the Swarthmore Spheromak Experiment (SSX). Magnetic reconnection is the dominant dynamical process at play while two spheromaks of equal but opposite sign magnetic helicity merge axially in the cylindrical SSX device. Typical conditions for the hydrogen plasmas produced by SSX are $T_{i}, T_{e} \approx 15-20 \mathrm{eV}, n_{e}$ $\approx 10^{14-15} / \mathrm{cm}^{3}, B \approx 1 \mathrm{kG}$, and the impurity fraction is of order $<1 \%$.

The design of the SSX IDS instrument incorporates an echelle diffraction grating and multianode photomultiplier tube (PMT) in order to achieve high spectral resolution and fast time response. The combination of these two components is not only unique to this IDS instrument but is also essential to its exceptional performance characteristics. This instrument can analyze spectral lines from the UV through

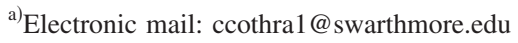

visible with resolution $R=\lambda / \delta \lambda=(1.8-3.5) \times 10^{4}$ [with $\delta \lambda$ the full width at half maximum (FWHM) of the instrument function, corresponding to an instrument temperature of 2-7 eV for C impurity ions] and is absolutely calibrated to better than $\pm 6 \mathrm{~km} / \mathrm{s}$. The SSX IDS instrument has been used initially to monitor the time dependence of the line shape of $\mathrm{C}$ III emission at $229.687 \mathrm{~nm}$ for the duration $(\approx 100 \mu \mathrm{s})$ of a spheromak merging experiment. The $1 \mu$ s time resolution of these measurements is fast enough to follow changes in flow and ion temperature at the characteristic magnetohydrodynamic (MHD) time scale of SSX plasmas.

\section{INSTRUMENT DESCRIPTION}

The components of the SSX IDS instrument are shown schematically in Fig. 1. The collection and input optics select a view chord through the plasma and efficiently couple light from this chord into the spectrometer. The multianode PMT is difficult to use in a high resolution spectrograph because of the relatively large $0.8 \mathrm{~mm}$ width and $1 \mathrm{~mm}$ spacing of its channels. Assisted by a modest $3.7 \times$ magnification from the output optics, the echelle grating spectrometer provides the large dispersion across the PMT necessary to overcome this difficulty. An echelle grating is conceptually no different from a standard grating; the only distinction is that the blaze angle is much larger and the groove spacing is more coarse for an echelle.

The design of the SSX IDS instrument targeted the C III $229.687 \mathrm{~nm}$ emission line, which previous spectroscopic studies had found to be strong in SSX plasmas. This line is observed at 25th order (echelle gratings naturally operate at large diffraction order) with dispersion of $0.0085 \mathrm{~nm} / \mathrm{mm}$ across the PMT. The resolution for the SSX IDS instrument at this setting is $R=2.5 \times 10^{4}$. The instrument temperature $k T_{\text {inst }} \equiv m_{i} c^{2}(\delta \lambda / \lambda)^{2} /(8 \ln 2)$ (i.e., the width of the instrument function interpreted as an ion temperature, in this case for $\mathrm{C}$ ions) is $3.4 \mathrm{eV}$. The bandwidth $(0.142 \mathrm{~nm})$ for the central 16 PMT channels currently in use corresponds to $\pm 92 \mathrm{~km} / \mathrm{s}$. Therefore, given $T_{i} \approx 15-20 \mathrm{eV}$ and Alfvén 


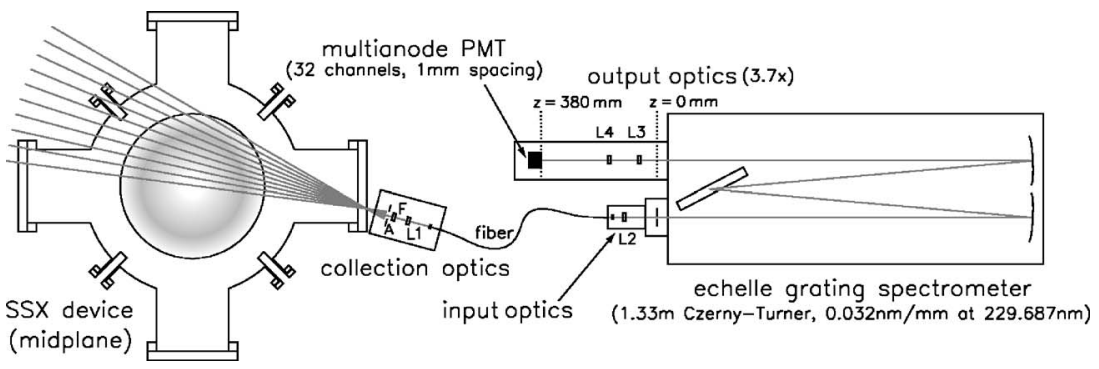

FIG. 1. The layout of the SSX IDS instrument. The collection optics, consisting of an aperture (A), interference filter (F), lens (L1), and optical fiber, can view any one of the ten view chords at the midplane of the cylindrical SSX device. Input optics (lens L2) efficiently couples the collected light into the echelle spectrometer, and output optics (lenses L3 and L4) image the spectrometer focal plane (at $z=0 \mathrm{~mm}$ ) with $3.7 \times$ magnification onto the multianode photomultiplier tube (at $z=380 \mathrm{~mm}$ ).

(MHD) speed $v_{A} \approx 100 \mathrm{~km} / \mathrm{s}$ typical of SSX plasmas, the thermally broadened C III $229.687 \mathrm{~nm}$ line is well resolved over several channels and the largest expected Doppler shifts $\left( \pm v_{A}\right)$ fit within the available bandwidth.

The SSX IDS instrument can analyze UV wavelengths from 200 to $270 \mathrm{~nm}$, and visible wavelengths from 330 to $650 \mathrm{~nm}$. These ranges are determined by the refractive output optical system (described in detail below), except the bound at $650 \mathrm{~nm}$ which is set by the sensitivity of the PMT photocathode. Figure 2(a) shows the correlation (determined by the grating equation, the optics of the spectrometer, and the characteristics of the echelle grating) between the central wavelength setting of the spectrometer and the grating angle for diffraction orders $n=9$ through $n=26$ (solid/ dotted lines) from the UV to the visible. The solid line segments in Fig. 2(a) indicate the settings at which the grating efficiency is largest; this condition holds at only one of the multiple grating angles (diffraction orders) at which a given wavelength can be observed. The grating efficiency is largest within the peak of the blaze function (the angular distribution of light diffracted by a single groove). This peak falls between the dashed curves which mark contours of the blaze function at $(\pi / 2)^{-2} \approx 40 \%$.

This sawtoothing of the grating angle as a function of the central wavelength setting typical of an echelle spectrometer design causes the same behavior in the spectral characteristics of the SSX IDS instrument. As shown in Fig. 2(b), the resolution varies from a minimum of $1.8 \times 10^{4}$ to a maximum of $3.5 \times 10^{4}$. For instrument temperature, the variation is $2-7 \mathrm{eV}$ (for $\mathrm{C}$ ions). Similarly, the anamorphic magnification varies from 1.3 to 1.6 , which means that the entrance slit width must be adjusted to keep its image at the PMT matched to the $0.8 \mathrm{~mm}$ channel size for optimal spectral resolution and throughput (the image of the entrance slit formed by the spectrometer is wider by a factor $d \beta / d \alpha$, known as the anamorphic magnification, where $\alpha$ and $\beta$ are, respectively, the angles of rays incident to and diffracted from the grating).

For the UV range of operation of the SSX IDS instrument, the collection, input, and output optical systems use a set of UV-grade fused silica (UVGFS) lenses. A mechanically identical (i.e., lens shape and diameter) set of BK7 lenses is used for the visible range. Because the refractive index for BK7 within the visible range of operation is numerically the same as for UVGFS within the UV range, these two sets of lenses are also optically identical. The focal lengths reported below for these lenses are computed for a refractive index of 1.52 (which is the index of UVGFS at the C III $229.687 \mathrm{~nm}$ design wavelength). Further detail of the SSX IDS instrument design is described in the following subsections.

\section{A. Collection and input optics}

A simple optical assembly consisting of an aperture, interference filter, lens, and optical fiber is used to collect light
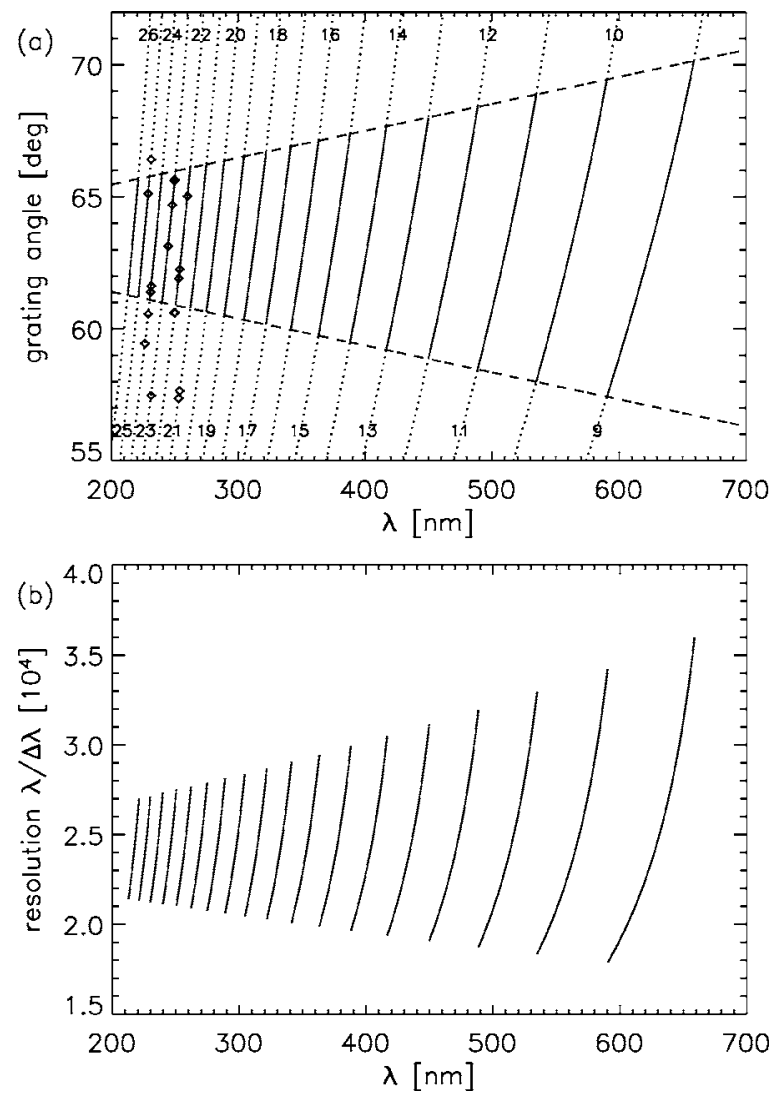

FIG. 2. (a) The operating space of the SSX IDS instrument. Solid/dotted lines indicate the correlation of wavelength and grating angle for diffraction orders $n=9$ through $n=26$. The grating efficiency is largest between the dashed lines, which mark the contours at which the blaze function is $(\pi / 2)^{-2} \approx 40 \%$. Data points indicate spectral lines observed during calibration studies. The sawtooth behavior of the grating angle is imprinted on the resolution (b) and other spectral characteristics of the SSX IDS instrument (a $1.0 \mathrm{~mm}$ instrument function FWHM is used for this calculation; the actual instrument function can vary with wavelength due to the chromatic aberration of the refractive output optics). 
from the plasma through a quartz vacuum window at the midplane of the cylindrical SSX device. The optics are mounted on a rotatable arm which may be positioned to select any one of ten different view chords (see Fig. 1).

The interference filter is necessary to attenuate any light coming from overlapping diffraction orders [as indicated in Fig. 2(a), there are multiple solutions to the grating equation for a given grating angle]. The filter used for observations of the C III $229.687 \mathrm{~nm}$ line has a center wavelength of $229.4 \mathrm{~nm}$, FWHM of $10 \mathrm{~nm}$, and peak transmission of $19 \%$; this and a similar filter with a center wavelength of $248.5 \mathrm{~nm}$ were used for calibration studies. Filters used for visible lines have larger peak transmissions (near 50\%). Out of band blocking is typically better than $10^{-4}$.

The planoconvex spherical lens (L1 in Fig. 1) has a $74.2 \mathrm{~mm}$ focal length and is $25.4 \mathrm{~mm}$ in diameter. The solarization-resistant (high OH) UVGFS multimode optical fiber has a $0.6 \mathrm{~mm}$ core diameter, 0.22 numerical aperture (NA), and $1 \mathrm{~m}$ length. Its transmission is nearly $100 \%$ for visible light and drops to about $80 \%$ in the UV.

The aperture, $15 \mathrm{~mm}$ in diameter, is located at the front focal point of the lens. The fiber is located $2.8 \mathrm{~mm}$ past the back focal point of the lens (i.e., the fiber is nearly focused at infinity). Only rays diverging at less than $f / 50$ from the optical axis of the view chord are collected. To a very good approximation, the diameter of the view chord is constrained to less than $15 \mathrm{~mm}$ throughout the plasma.

The fiber is filled at $f / 5$. Because the fiber has a large core diameter, is short, and is kept fairly straight, this $f$ number is preseved at the exit. Any spatial variation of intensity at the input, however, is effectively homogenized by the multiple internal reflections along the length of the fiber. Lens L2 of the input optics section in Fig. 1 is a $24.2 \mathrm{~mm}$ focal length spherical biconvex lens which images the output end of the fiber onto the entrance slit with $2 \times$ magnification. Light thus enters the spectrometer at $f / 10$, slightly overfilling the spectrometer acceptance (see below).

\section{B. Spectrometer}

The SSX IDS instrument is built around a McPherson Model 209 spectrometer. This is a $1.33 \mathrm{~m}$ focal length Czerny-Turner spectrometer with spherical collimating and focusing mirrors ( $\mathrm{Al}-\mathrm{MgF}_{2}$ coated) and optimized for spectral resolution. The most important modification to this spectrometer is the installation of an echelle plane grating. The echelle is ruled at $316 / \mathrm{mm}$ groove density with a blaze angle $\theta_{b}=63.43^{\circ}$ (it is an R2 grating, meaning $\tan \theta_{b}=2$ ). The manufacturer did not characterize the grating efficiency; however, typical efficiencies for echelle gratings peak at more than $50 \%$, but somewhat less than for standard gratings $(\approx 80 \%)$, with angular dependence as described in the previous section.

The height of the grating is standard at $110 \mathrm{~mm}$ while the width is oversized at $220 \mathrm{~mm}$; the ruled area is 104 $\times 204 \mathrm{~mm}^{2}$ Vertically, the aperture of the instrument is about $f / 12$. The optical design of the model 209 is optimized to fill a standard grating (with longer grooves) and has $f / 9.4$. Thus, despite the large width of the echelle grating, it is not quite large enough to fully intercept the beam the collimating mirror can deliver. This geometric effect is due to the large incidence angle (near $\theta_{b}$ ) of the collimated beam on the grating. Consequently, the effective aperture in the horizontal direction is also approximately $f / 12$.

A mechanical counter geared to the sine bar gives wavelength readout with approximately $1 \times 10^{-5}$ precision. The grating angle can be adjusted manually or by a stepper motor controller (for greater precision). The grating is mounted on the turntable at an angular offset of $30^{\circ}$ in order to access the large angles needed for operation of the echelle.

For observation of the C III $229.687 \mathrm{~nm}$ line, the entrance slit of the spectrometer was set to $150 \mu \mathrm{m}$. Given the anamorphic magnification (1.48) at this setting and output optics magnification, the image of the slit at the PMT should be $0.82 \mathrm{~mm}$ wide, which is well matched to the channel width of the PMT for optimal wavelength resolution and throughput; however, as described in Sec. III, the measured width of the instrument function is larger than this. The slit height was $1 \mathrm{~mm}$, which slightly cropped the $1.2 \mathrm{~mm}$ diameter image of the fiber produced by the input optics (the slit mask has a maximum height of $20 \mathrm{~mm}$ ). In general, a Czerny-Turner spectrometer forms a curved image of the entrance slit; however, this effect contributes negligibly to the wavelength resolution due to the short slit height used.

\section{Output optics}

The output optical system forms an inverted image of the spectrometer focal plane on the multianode PMT with $3.7 \times$ magnification. Two planoconvex spherical lenses are used, labeled L3 and L4 in Fig. 1. L3 is a $49.6 \mathrm{~mm}$ focal length lens and L4 is a $99.0 \mathrm{~mm}$ focal length lens. With the spectrometer focal plane (object plane) defining $z=0$, the detector focal plane (image plane) is formed at $z=380 \mathrm{~mm}$, as indicated in Fig. 1. The flat lens surfaces are located at $z$ $=24.8 \mathrm{~mm}$ and $z=122.6 \mathrm{~mm}$, respectively. Both flat surfaces face the spectrometer for sharpest focusing.

The chromatic aberration of the output optics limits the useful wavelength range of the SSX IDS instrument in the UV. Ray trace studies indicate that the reduction in spectral resolution due to poor imaging is acceptable over the range $1.505<n<1.540$ of refractive index when the PMT is fixed at $z=380 \mathrm{~mm}$ (the imaging is best at this position for $n$ $=1.52$, by construction). At the extrema of this range, the instrument temperature is $50 \%$ larger than the instrument temperature for $n=1.52$ (where the instrument function is triangular). The wavelength interval corresponding to this range of refractive index for UVGFS is $209-256 \mathrm{~nm}$.

The PMT is mounted on a two axis translation stage which permits $\Delta z= \pm 12.5 \mathrm{~mm}$ of motion along the optical axis to reduce the chromatic defocusing (the orthogonal motion of the stage is in the dispersive direction). Although this range in $z$ is not large enough to fully correct the imaging over the 209-256 nm range, adjustment of the position of the PMT holds the increase in instrument temperature to less than $11 \%$. With the PMT at the $\pm 12.5 \mathrm{~mm}$ limits of its motion, the increase in the instrument temperature is at most $50 \%$ for the wavelength interval of $200-270 \mathrm{~nm}$. This loss of resolution can be corrected by narrowing the entrance slit width (by up to half), if decreased throughput is acceptable. 
For the BK7 lenses, the refractive index range given above corresponds to wavelengths from the infrared to about $330 \mathrm{~nm}$. The transmission of BK7 falls sharply at this short wavelength limit. For the IDS system as a whole, the phototube sensitivity sets the long wavelength limit at $650 \mathrm{~nm}$ (see below). The chromatic aberrations are therefore much more of a problem for the operation of the SSX IDS instrument in the UV than in the visible.

\section{Multianode photomultiplier tube}

The detector used in the SSX IDS instrument is a Hamamatsu H7260A-03 multianode photomultiplier tube. This tube has a bialkali photocathode on a UVGFS window and an array of 32 separate dynode chains spaced with $1 \mathrm{~mm}$ pitch; each channel is sensitive to a $0.8 \mathrm{~mm}$ wide by $7 \mathrm{~mm}$ tall section of the photocathode. The channel-to-channel response varies by as much as $17 \%$, and up to $3 \%$ crosstalk with neighboring channels is typical. Among the central 12 channels used for measurements of the C III $229.687 \mathrm{~nm}$ line, the response uniformity is $9 \%$.

This tube is sensitive from 185 to $650 \mathrm{~nm}$ with peak quantum efficiency of $20 \%$ at $420 \mathrm{~nm}$. For the C III $229.687 \mathrm{~nm}$ line the quantum efficiency is about $5 \%$. At $-800 \mathrm{~V}$, the gain is $5 \times 10^{6}$. The time response is very fast: the single photoelectron pulse has a $0.6 \mathrm{~ns}$ rise time and is 2 ns wide. For each channel, nonlinearity is less than $\pm 2 \%$ for pulses of up to $600 \mu \mathrm{A}$.

\section{CALIBRATION STUDIES}

The absolute wavelength calibration, dispersion, and instrument function shape are the most important instrument characteristics affecting the accuracy of the spectroscopic Doppler velocity and temperature measurements. Line radiation from $\mathrm{B}, \mathrm{Cd}$, and $\mathrm{Sb}$ metal vapor hollow cathode lamps (Hamamatsu L233) and from a $\mathrm{Hg}$ pen lamp were used to evaluate these properties of the SSX IDS instrument.

The cluster of data points in Fig. 2(a) in the wavelength range of 226-260 $\mathrm{nm}$ indicates the spectral lines used for the calibration studies. A total of 11 lines are observed, some at multiple orders (there are 18 different values of $n \lambda$ ). This data set largely covers the operating range in grating angle, except at the largest grating angles which are only required for the longest anticipated wavelengths.

With the SSX IDS instrument operating as a scanning monochrometer (only using the PMT channel closest to the optical axis), the mechanical wavelength counter reading corresponding to the peak response for each spectral line was recorded, thus yielding the absolute wavelength calibration shown in Fig. 3. The fractional error in the counter is evidently systematic and well described by a linear fit over the required operating range in grating angle. After correcting for this linear dependence, the residual is less than \pm 2 $\times 10^{-5}$ or about $\pm 6 \mathrm{~km} / \mathrm{s}$. When observing the $\mathrm{C}$ III $229.687 \mathrm{~nm}$ at 25 th order, the absolute wavelength calibration error corresponds to $\pm 0.009 \mathrm{~nm}$. Since the scatter about the fit is much greater than the error in the data, the origin of the remaining error must also be systematic.

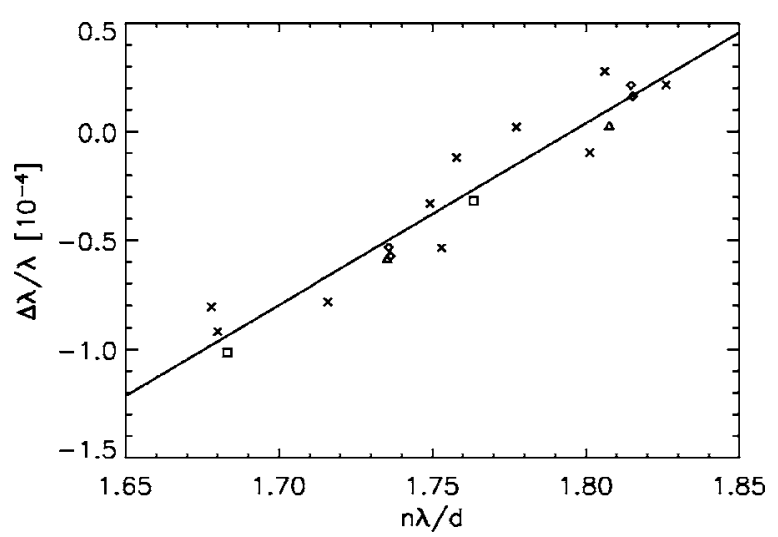

FIG. 3. The fractional error $\Delta \lambda / \lambda$ of the mechanical wavelength counter measured at the spectrometer settings indicated in Fig. 2(a) for the calibration spectral lines; the quantity $n \lambda / d$, where $d$ is the groove spacing, depends only on the grating angle. The linear correlation is easily corrected, yielding a residual absolute wavelength calibration error of less than $\pm 6 \mathrm{~km} / \mathrm{s}$. Error bars (not shown) are about the same size as the plotting symbols.

Figure 4 shows the instrument functions (thin lines) measured for channels located on the optical axis $(x=0)$ and at $x= \pm 7 \mathrm{~mm}$ (where $x$ is the dispersive coordinate). These were obtained by scanning the grating angle to sweep the $231.147 \mathrm{~nm} \mathrm{Sb}$ line at 25th order across the PMT (the image of the $0.150 \mathrm{~mm}$ wide entrance slit should be $0.82 \mathrm{~mm}$ on the PMT at this setting). For direct comparison, each instrument function has been centered on its peak. Apparently, all channels share the same instrument function shape. This is not quite the triangular shape anticipated for a rectangular entrance slit image matched to the width of the PMT channel. Furthermore, the $1.06 \mathrm{~mm}$ FWHM is significantly wider than the $0.82 \mathrm{~mm}$ expected width; nevertheless, this value has been used for computing the instrument temperature and resolution of the SSX IDS instrument.

The lines of the B hollow cathode lamp at 249.677 and $249.773 \mathrm{~nm}$ are spaced closely enough to fit within the 23rd order bandwidth of the SSX IDS instrument. With the spectrometer set for $249.725 \mathrm{~nm}$ (the average of these two wavelengths), the separation of these lines at the PMT is found to

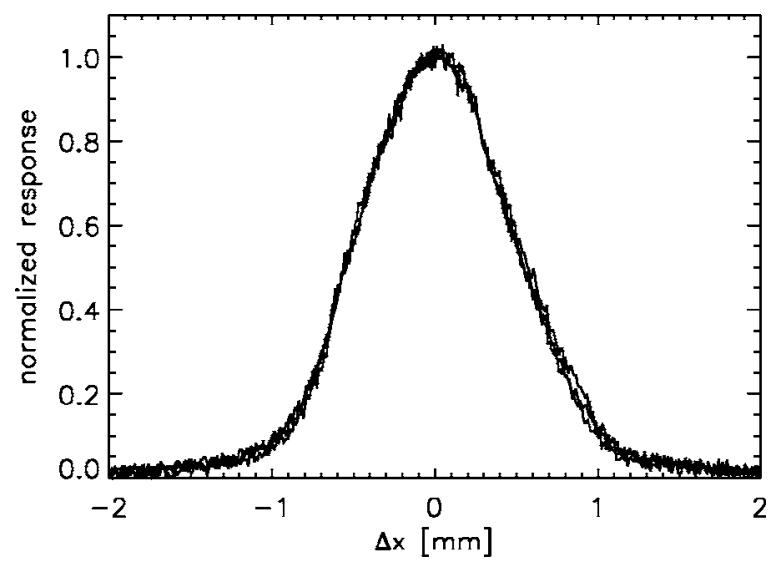

FIG. 4. The instrument function for three different channels, located on the optical axis $(x=0)$ and at $x= \pm 7 \mathrm{~mm}$. Each instrument function has been centered about the location of its peak; evidently, all three have the same shape. 


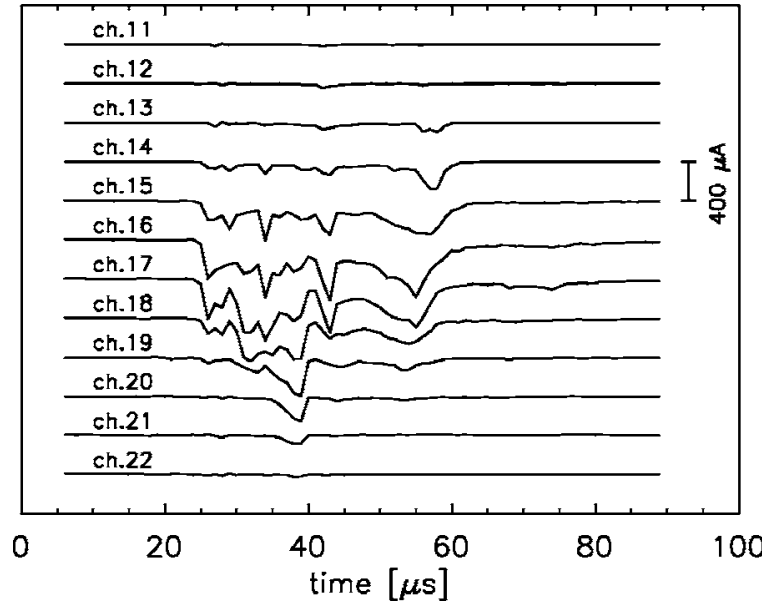

FIG. 5. Signals from the central 12 channels of the multianode PMT measured during a counterhelicity spheromak merging experiment.

be $10.38 \pm 0.05 \mathrm{~mm}$. The dispersion is therefore directly measured to be $(9.25 \pm 0.05) \times 10^{-3} \mathrm{~nm} / \mathrm{mm}$ at this setting. The ratio of this measured dispersion at the detector focal plane to the dispersion at the spectrometer focal plane (computed to be $0.0343 \mathrm{~nm} / \mathrm{mm}$ ) gives a value of 3.70 for the magnification of the output optics, in excellent agreement with the value obtained from the ray trace simulations. This selfconsistency suggests that the computed dispersion for other settings may be used with great confidence.

Specifically for measurement of the C III $229.687 \mathrm{~nm}$ line at 25th order, the absolute wavelength calibration is extremely accurate. At this spectrometer setting, the $249.677 \mathrm{~nm}$ line from the B lamp happens to fall within the instrument bandwidth (in fact, the overlapping 23rd order central wavelength is $249.660 \mathrm{~nm}$, so the B line appears displaced off the optical axis by only $1.84 \mathrm{~mm}$ ). The location of this line on the PMT therefore specifies the absolute wavelength calibration of the detector focal plane. As for the dispersion measurement, this location is measured by scanning the position of the PMT (i.e., the grating angle is fixed) and is determined to within $\pm 0.05 \mathrm{~mm}$, which is equivalent to $\pm 0.6 \mathrm{~km} / \mathrm{s}$ error in the absolute wavelength calibration. This is comparable to the fractional variation in wavelength due to changes in the refractive index of air from ambient temperature or pressure fluctuations.

\section{RESULTS}

The SSX IDS instrument has been used initially to analyze the C III $229.687 \mathrm{~nm}$ emission line during counterhelicity spheromak merging experiments. The configuration and spectral characteristics of the SSX IDS for these measurements are the same as has been described in the previous sections. As expected, this line is very bright. The PMT was operated at a reduced gain of $7 \times 10^{5}(-650 \mathrm{~V})$ to avoid nonlinearity, and the signals were conditioned with $1 \mu \mathrm{s}$ time constant averaging.

Figure 5 shows the time dependent response of the central 12 channels during a single discharge of the SSX device. Two examples of the line shapes constructed from these data are displayed in Fig. 6; the dotted lines are Gaussian fits.
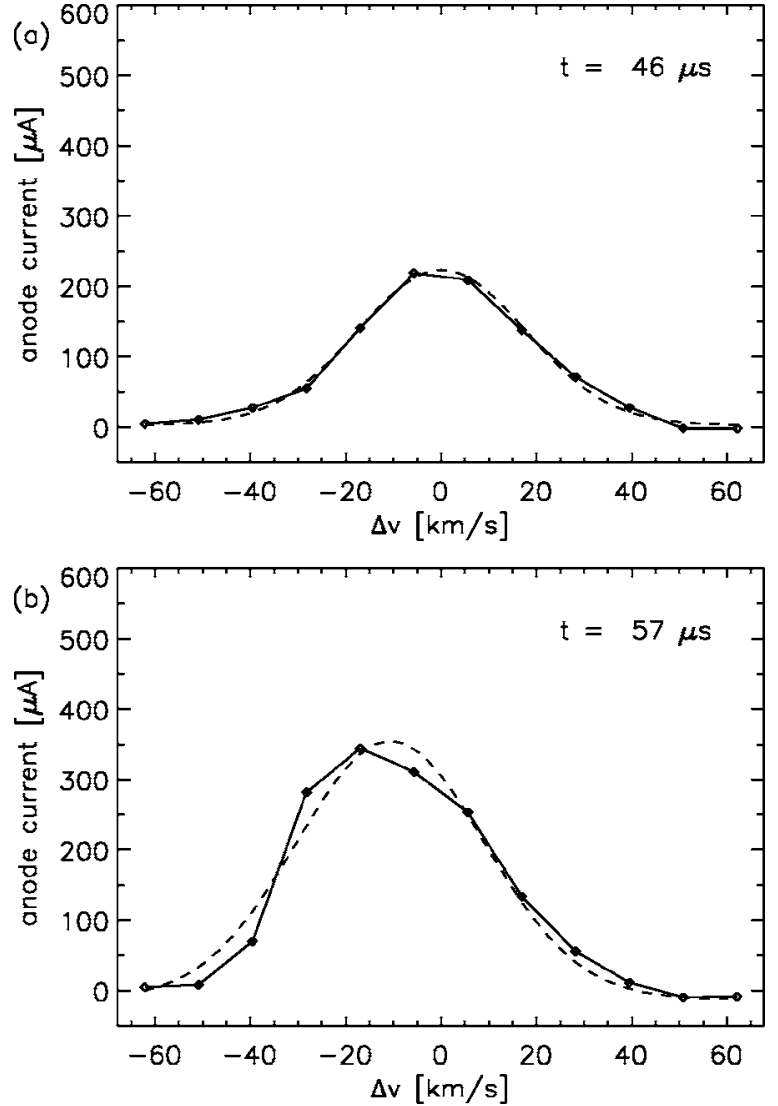

FIG. 6. Line shapes constructed from the data of Fig. 5 at (a) $t=46 \mu$ s and at (b) $t=57 \mu \mathrm{s}$. Dotted lines show Gaussian fits; the data in (b) clearly indicate a non-Maxwellian line shape. Note that no background subtraction has been applied to this data.

Like the data of Fig. 6(b), the line shapes typically indicate some deviation from a Gaussian. There are many reasons why a non-Gaussian line shape would be observed, including local variation of ion temperature, fluid velocity, and emissivity along the view chord, as well as plasma kinetic effects producing an inherently non-Maxwellian velocity space distribution. Detailed discussion of these issues, however, is outside the scope of this article.

Setting these concerns aside, the time dependence of the Doppler shift and thermal width extracted from the data of Fig. 5 are shown in Fig. 7. The shift and width are obtained both from the first and second moments of the line shapes as well as from the parameters for the Gaussian fit. Because the absolute wavelength calibration for this setting is so precise, the error in the measured shift is essentially entirely due to the error in determining the centroid of the line shape (first moment or mean of the Gaussian), which depends in general on the width of the line shape and the signal to noise ratio in each channel (which improves with increasing line strength).

Formal deconvolution of the instrument function from the observed line shapes to obtain the intrinsic spectral line shape was not performed for the determination of the ion temperature shown in Fig. 7(b). Instead, the widths were assumed to add in quadrature (which is correct only when convoluting two Gaussians), so the instrument temperature was merely subtracted from the temperature of the observed line shape. Because the instrument temperature is much 

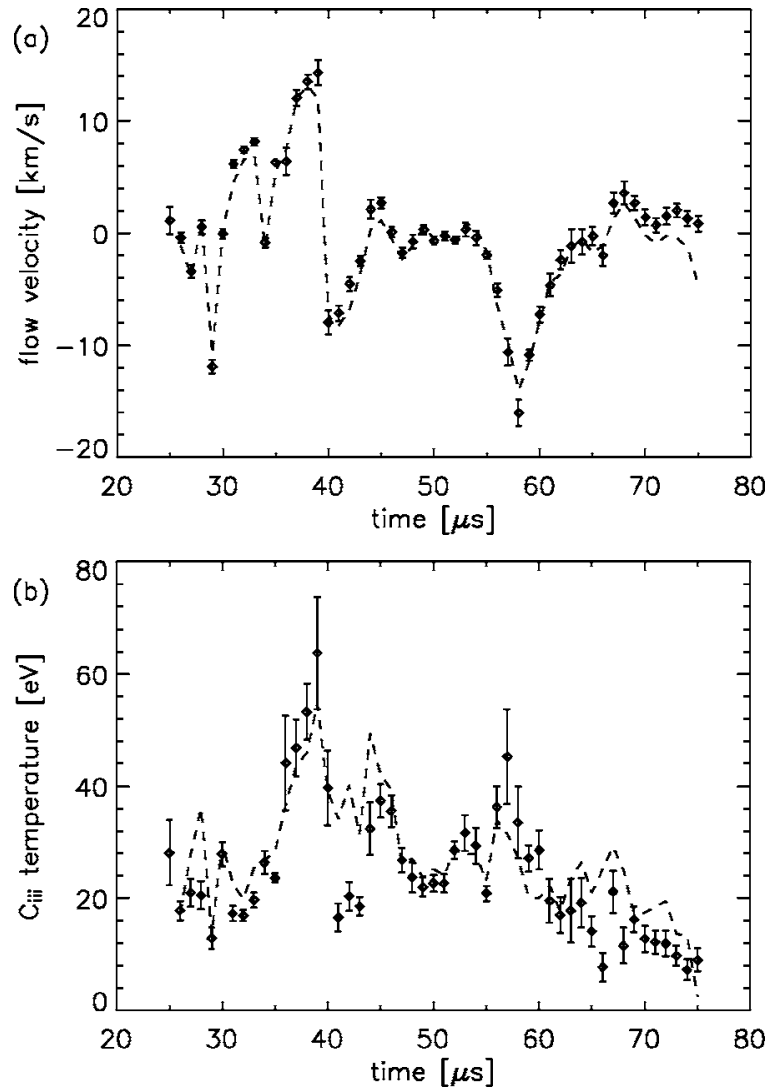

FIG. 7. The Doppler shift (a) and thermal width (b) derived from the data of Fig. 5. The data points with error bars are obtained from a Gaussian fit to the line shapes. Analysis of the first and second moments of the line shapes yields the results indicated by the dashed lines.

smaller than $T_{i}$, the error from this approximation should be small. No correction is necessary for Stark broadening, ${ }^{11,12}$ which is found to be negligible for the SSX plasma conditions (the equivalent temperature is less than $0.2 \mathrm{eV}$ ). The classical collisional slowing down time and thermal equilibration time between $\mathrm{C}$ ions and the majority protons are both less than $0.5 \mu \mathrm{s}$. Therefore, the $\mathrm{C}$ ions are effectively at the same temperature and moving with the same velocity as the majority species at the time resolution $(1 \mu \mathrm{s})$ of the measurement. Barring more complicated kinetics, these IDS results may be interpreted as a measurement of the fluid velocity and majority ion (proton) temperature of SSX plasmas.

\section{DISCUSSION}

The most severe constraint on the SSX IDS instrument design is the requirement that the time dependence of the line shape be monitored with good time resolution. Using the SSX plasma radius as a characteristic scale length $L$ $=20 \mathrm{~cm}$, the MHD time scale is $L / v_{A} \approx 2 \mu \mathrm{s}$. Of all detector array technologies, only the multianode PMT offers the combination of gain and time response required for this application. However, the $1 \mathrm{~mm}$ spatial resolution of each channel, although indeed remarkable for a PMT, is about 50 times larger than the pixel size of solid-state detectors such as charge-coupled devices (CCDs) or photodiode arrays com- monly used for high resolution spectroscopy. To achieve the same wavelength resolution, the dispersion at the PMT must therefore be 50 times greater.

The echelle grating in the SSX IDS design compensates for the large channel size of the multianode PMT by dramatically increasing the dispersion compared to a standard grating spectrometer design. For an echelle in a Czerny-Turner mounting, both the incident angle $\alpha$ and diffraction angle $\beta$ are large (i.e., close to the blaze angle $\theta_{b}$ ) to remain within the single groove diffraction peak (blaze function) where the grating efficiency is largest [see Fig. 2(a)]. From the grating equation, $n \lambda=d(\sin \alpha+\sin \beta)$, where $n$ is the diffraction order and $d$ is the groove spacing, the linear dispersion for an instrument with focal length $f$ is

$$
\frac{d \lambda}{d x}=\left(\frac{\lambda}{f}\right) \frac{\cos \beta}{\sin \alpha+\sin \beta} .
$$

Clearly, greater dispersion (numerically smaller $d \lambda / d x$ ) is obtained for larger $\alpha$ and $\beta$. Given a detector width $\delta x$ and assuming optimal wavelength resolution (triangular instrument function), the resolution may be expressed as

$$
R=\left(\frac{f}{\delta x}\right) \frac{\sin \alpha+\sin \beta}{\cos \beta} .
$$

Sufficient resolution is readily achieved through the $f / \delta x$ factor for solid-state detectors without ever needing to consider the dependence on $\alpha$ and $\beta$. The SSX IDS echelle grating design exploits this angular dependence to achieve high spectral resolution despite the large channel width $\delta x$ of the multianode PMT.

This design optimization for a large detector simultaneously leads to a spectrometer with larger étendue (product of entrance slit area and accepted solid angle), provided there are no constraints on the slit height. As described previously, the entrance slit should be wide enough that its image on the detector is the same size as the channel width $\delta x$. This yields a triangular instrument, function, the best wavelength resolution, and largest throughput; a wider slit clearly degrades the resolution, while a narrower slit simply analyzes less light. The large detector size allowed by an echelle design therefore also requires a wide entrance slit, which means large étendue. This is particularly important for time dependent measurements for which the time resolution can be limited by photon statistics.

Note, however, that the étendue of the SSX IDS instrument, in particular, as implemented with a $1 \mathrm{~mm}$ slit height, is not significantly larger than can be achieved (at the same resolution) using a standard grating spectrometer with larger $f$ number and using a much narrower but taller slit and a CCD (ignoring the issue of time response) placed directly at the spectrometer focal plane. Significant headroom is available, however, should more signals be required. The $7 \mathrm{~mm}$ height of the photocathode is not currently being fully illuminated with the $1 \mathrm{~mm}$ slit height. A $2 \mathrm{~mm}$ tall slit and a different input coupling (e.g, a linear fiber bundle) will about double the throughput and fill the active area of the PMT. A straightforward but more elaborate modification of the output optics using cylindrical lenses to provide unity magnification 
vertically and the necessary $3.7 \times$ magnification in the dispersive direction would permit a slit height of up to $7 \mathrm{~mm}$.

Some trade-offs limit the advantages of an echelle design (see Schroeder ${ }^{13}$ for a detailed discussion). Although the étendue may increase significantly, the throughput may not due to the need for an interference filter, as in the SSX IDS instrument, to eliminate light from overlapping orders. Filter transmission can be quite good $(>50 \%)$ for visible wavelengths, but in the UV the peak transmission drops to about $20 \%$. In addition, the large incident angle for echelle operation means that the width of the grating must be quite large to intercept the full collimated beam; some étendue is clearly lost (the effective $f$ number of the spectrometer increases) if the grating is not large enough. Another potential drawback is the modulation of the grating efficiency over a large wavelength range [see Fig. 2(a)], which could be undesirable if it is necessary to compare the absolute intensity of several lines.

Use of the echelle grating in the SSX IDS instrument does not completely compensate for the large channel width of the multianode PMT to achieve the desired resolution. As described above, the dispersion at the PMT is increased by the $3.7 \times$ magnification of the output optics (the detector size $\delta x$ relevant to the discussion above is thus $0.270 \mathrm{~mm}$, i.e., the effective channel width at the spectrometer focal plane). In other IDS instruments using a multianode PMT, ${ }^{8,9}$ output optics with large magnification power (more than $30 \times$ ) is the only compensation for the large detector size. The large magnification is necessary to produce the required dispersion horizontally, but the distribution of light vertically is much taller than the PMT entrance window, and much of the available photon flux is wasted. Furthermore, for such large magnifications, it is difficult to maintain good imaging off-axis, potentially causing degraded resolution and nonlinearity in the dispersion across the detector. This is to be contrasted to the SSX IDS instrument which exploits the optical properties of the echelle spectrometer to accomplish most of the compensation for the large channel size of the multianode PMT, thus placing modest requirements upon the output optics.

\section{SUMMARY}

A high resolution, fast time response echelle spectrograph has been constructed and used for passive Doppler spectroscopy of line radiation from impurity ions in SSX plasmas. The SSX IDS instrument can analyze spectral lines from the UV through visible with resolution ranging from $1.8 \times 10^{4}$ to $3.5 \times 10^{4}$. The instrument temperature is $2-7 \mathrm{eV}$ for $\mathrm{C}$ impurity ions, and the absolute wavelength calibration (i.e., the "zero velocity" location for Doppler shift measurements) is better than $\pm 6 \mathrm{~km} / \mathrm{s}$. The SSX IDS instrument has been used to measure the shape of the $229.687 \mathrm{~nm}$ emission line from C III impurity ions in SSX plasmas for the duration $(\approx 100 \mu \mathrm{s})$ of a counterhelicity spheromak merging experiment; these measurements are fast enough $(1 \mu$ s time resolution) to track changes in the line-averaged fluid velocity and ion temperature occurring at the MHD time scale of SSX plasmas. For this setting, the bandwidth of the 16 channels currently in use is $\pm 92 \mathrm{~km} / \mathrm{s}$.

The echelle grating and multianode PMT are the critical components in the unique design of the SSX IDS instrument. These two components complement one another very well for the purpose of high resolution, fast time response spectroscopy. By taking advantage of the extreme dispersion available with an echelle grating, high spectral resolution is attained despite the relatively wide $(0.8 \mathrm{~mm})$ channels of the PMT. Furthermore, because the slit width in any spectrometer design is matched to the detector width (after accounting for the anamorphic magnification), the echelle design can have large étendue. Although this instrument was developed for a laboratory plasma experiment, the design can easily be adapted to other applications with similar performance requirements.

\section{ACKNOWLEDGMENTS}

The authors wish to thank E. Schoeffel (McPherson, Inc.) and D. Craig and D. Den Hartog (U. Wisconsin) for useful discussions at the inception of this project. This work was supported by grants from the Department of Energy and the National Science Foundation.

${ }^{1}$ I. H. Hutchinson, Principles of Plasma Diagnostics (Cambridge University Press, Cambridge, 2002).

${ }^{2}$ J. Ghosh, R. C. Elton, H. R. Griem, A. Case, R. Ellis, A. B. Hassam, S. Messer, and C. Teodorescu, Phys. Plasmas 11, 3813 (2004).

${ }^{3}$ R. F. Ellis et al., Phys. Plasmas 12, 055704 (2005).

${ }^{4}$ U. Shumlak, B. A. Nelson, R. P. Golingo, S. L. Jackson, E. A. Crawford, and D. J. Den Hartog, Phys. Rev. Lett. 87, 205005 (2001).

${ }^{5}$ D. J. Den Hartog and R. P. Golingo, Rev. Sci. Instrum. 72, 2224 (2001).

${ }^{6}$ D. J. Den Hartog and R. J. Fonck, Rev. Sci. Instrum. 65, 3238 (1994).

${ }^{7}$ E. Scime, M. Cekic, D. J. Den Hartog, S. Hokin, D. J. Holly, and C. Watts, Phys. Fluids B 4, 4062 (1992).

${ }^{8}$ H. S. McLean et al., Rev. Sci. Instrum. 72, 556 (2001).

${ }^{9}$ P. Gu, M. Nagata, K. J. McCollam, T. R. Jarboe, B. A. Nelson, and A. J. Redd, Rev. Sci. Instrum. 75, 1337 (2004).

${ }^{10}$ C. D. Cothran, A. Falk, A. Fefferman, M. Landreman, M. R. Brown, and M. J. Schafffer, Phys. Plasmas 10, 1748 (2003).

${ }^{11}$ N. Konjević, A. Lesage, J. R. Fuhr, and W. L. Wiese, J. Phys. Chem. Ref. Data 31, 819 (2002).

${ }^{12}$ H. R. Griem, Principles of Plasma Spectroscopy (Cambridge University Press, New York, 1997).

${ }^{13}$ D. J. Schroeder, Astronomical Optics (Academic, New York, 1987). 